\title{
ANÁLISE ECONÔMICA DE UM PLANTIO DE TECA SUBMETIDO A DESBASTES ${ }^{1}$
}

\begin{abstract}
Antonio de Arruda Tsukamoto Filho², Márcio Lopes da Silva ${ }^{3}$ Laércio Couto ${ }^{3}$ e Marcelo Dias Müller ${ }^{2}$
RESUMO - Neste trabalho estudou-se a viabilidade econômica de um plantio de teca (Tectona grandis L.f.) submetido a desbastes. Foram utilizados os seguintes critérios de análise econômica de projetos: valor presente líquido, benefício (custo) periódico equivalente, taxa interna de retorno e valor esperado da terra. A taxa de desconto utilizada foi de $10 \%$ a.a. Os indicadores demonstraram a viabilidade econômica do plantio da teca, conforme as condições estabelecidas na análise. O plantio será inviável se houver redução maior que $25 \%$ nas receitas ou aumento superior a 35\% nos custos, sendo, portanto, mais sensível às variações na redução de receitas que às variações no aumento de custos.
\end{abstract}

Palavras-chave: Tectona grandis, avaliação econômica e plantação florestal.

\section{ECONOMIC ANALYSIS OF A TEAK PLANTATION SUBMITTED TO THINNING}

\begin{abstract}
This work evaluated the economic viability of a teak plantation (Tectona grandis L.f.) submitted to thinning. The following economic criteria of project analysis were applied: Net Present Value, Equivalent Annual Benefit (Cost), Internal Return Rate and Land Expected Value. The discount rate applied was 10\% a year. The indicators showed the economic viability of the teak plantation, under the conditions established in the analysis. The plantation will not be viable by reducing $25 \%$ of the revenues or increasing $35 \%$ of the costs, being more sensitive to the variations in income decrease than to variations in cost increase.
\end{abstract}

Key words: Tectona grandis, economic evaluation and forest plantation.

\section{INTRODUÇÃO}

A teca (Tectona grandis L.f.), também conhecida por teak (Índia, Siam, Birmânia e Indonésia), teck (França), ojati (Java), may sak (Laos) e tiek (Alemanha), é uma espécie arbórea da família Verbenaceae que apresenta alto valor comercial. O principal produto desta espécie é a madeira, muito utilizada na carpintaria, na marcenaria, na produção de peças de usos nobres e de móveis finos e, especialmente, na indústria da construção naval, onde é praticamente insubstituível, pelo fato de resistir ao sol, ao calor, ao frio e à água de chuvas e do mar (Rondon Neto et al., 1998). A combinação de beleza, resistência e durabilidade fez da madeira desta espécie uma das mais valiosas do mundo, superando outras madeiras nobres, como a do mogno (Swietenia macrophylla King).

Essa espécie ocorre naturalmente no centro e sul da Índia, em Myanmar, no norte da Tailândia e em Laos, atingindo altura de $35 \mathrm{~m}$ e DAP maior que $100 \mathrm{~cm}$. A teca foi introduzida em muitos países do sudeste asiático e alguns da África e das Américas. No Brasil, os plantios de teca iniciaram-se no final da década de 60, implantados pela empresa Cáceres Florestal S.A., na região do município de Cáceres - Mato Grosso, onde as condições climáticas são semelhantes às dos países de origem da espécie. Além das condições climáticas favoráveis, o solo

1 Recebido para publicação em 19.2.2002.

Aceito para publicação em 9.9.2003.

2 Doutorando Ciências Florestais, Dep. Engenharia Florestal da Universidade Federal de Viçosa - UFV, 36570-000 ViçosaMG, <tsukamoto@vicosa.ufv.br>; ${ }^{3}$ Prof. do Dep. de Engenharia Florestal-UFV. 
de melhor fertilidade e os tratos silviculturais mais adequados e intensos contribuíram para reduzir o ciclo de produção de 80 anos, na região de origem da teca, para apenas 25 anos, na região de Cáceres-MT.

No momento, o reflorestamento com teca no Brasil surge como uma ótima opção de investimento. Segundo Finger et al. (2001), a produção mundial de madeira de teca é estimada em 3 milhões de $\mathrm{m}^{3} / \mathrm{ano}$, o que é extremamente baixa pela demanda atual dessa espécie no mercado exterior. Veit (2000) afirmou que o desequilíbrio entre a oferta e a procura determinou a continuada valorização da madeira de teca, cujo preço registrou um ganho médio de $8,32 \%$ a.a., em dólar norteamericano, entre 1970 e 1999. Em Myanmar, toras de teca para faqueação (veneer quality) de Fourth Quality atingiu o valor médio de US\$3594/t.50p3 (dólar norteamericano por tonelada de 50 pés cúbicos, devendo ser ressaltado que 1 tonelada de $50 \mathrm{p} 3$ corresponde a $1,8 \mathrm{~m}^{3}$, medida geométrica) e toras para serrar (sawing quality) do tipo "Grade 1" atingiu o valor médio de US\$2367/ t.50p3.

Apesar desse potencial, os reflorestamentos de teca no Mato Grosso ainda não ultrapassam 20.000 ha. Também são poucas as empresas que estão investindo nas plantações florestais desta espécie, nesse Estado e no Brasil. Além disto, estudos econômicos dos plantios de teca no País praticamente não existem ou são de domínio apenas das empresas reflorestadoras. Uma análise dessa natureza será muito importante e trará contribuições para o setor florestal, podendo auxiliar na tomada de decisão acerca do planejamento, da administração e do retorno financeiro do reflorestamento de teca.

Nesse contexto, o objetivo deste trabalho foi realizar uma análise econômica de um plantio de teca (Tectona grandis L.f.) submetido a desbastes, implantado em área de cerrado no Estado de Mato Grosso.

\section{MATERIAL E MÉTODOS}

O presente estudo teve como fonte de dados os plantios florestais de teca implantados no Estado de Mato Grosso. Pela dificuldade de conseguir informações, alguns custos utilizados nesta análise foram estimados e outros obtidos através de valores médios calculados para plantios de eucalipto, guardadas as devidas diferenças entre as espécies quanto às atividades silviculturais e ao manejo da floresta.

\subsection{Estrutura de Custos}

Os custos das operações florestais relacionados às atividades de produção de mudas, implantação, manutenção, desbaste e exploração estão descritos no Quadro 1. Todos os custos fixos foram contemplados nesta análise, sendo considerados de acordo com a atividade desenvolvida.

Quadro 1 - Custos, em reais por hectare, das operações florestais para produção de mudas, implantação, manutenção, desbaste e exploração de teca, plantada no espaçamento $3 \times 2 \mathrm{~m}$

Table 1 - Costs, in Real, per hectare of forest operations for seedling production, establishment, maintenance, thinning and exploration of teak, planted in a $3 \times 2 \mathrm{~m}$ the spacing

\begin{tabular}{|c|c|c|}
\hline Especificação & Ano de Ocorrência & Custo Total (R\$/ha) \\
\hline Custos do projeto & 0 & 410,00 \\
\hline Custos de produção de mudas & 0 & 189,70 \\
\hline Custos de preparo do terreno & 0 & 301,50 \\
\hline Custos de plantio & 0 & 133,90 \\
\hline Custos de manutenção ( $1^{\circ}$ ano) & 1 & 215,50 \\
\hline Custos de manutenção ( $2^{\circ}$ ao $4^{\circ}$ ano $)$ & $2-4$ & 151,50 \\
\hline Custos de manutenção (desrama) & $2-5$ & 40,00 \\
\hline Custos de manutenção (adubação) & $4,8,12$ & 18,90 \\
\hline Custos anuais de manutenção & $1-25$ & 132,50 \\
\hline Custos de desbastes & $4,8,12,16,20$ & [Volume $\left.\left(\mathrm{m}^{3}\right) \times 127,1\right]^{*}$ \\
\hline Custos de exploração & 25 & [Volume $\left.\left(\mathrm{m}^{3}\right) \times 125,0\right]^{*}$ \\
\hline
\end{tabular}

* O fator de cubicação usado foi de 3,31 metros estéreo para cada metro cúbico de madeira retirada do plantio. 
Para realização do projeto foram considerados como custos de infra-estrutura (custo do projeto) a elaboração do projeto, os serviços de topografia, a construção de cercas, estradas e aceiros e o combate inicial às formigas.

Para obter o custo de produção de mudas, foram considerados os custos com coleta de sementes, semeadura no canteiro, condução e manutenção do viveiro, preparo dos tocos e administração (incluindo custo da terra). Estes custos foram baseados na produção de 6.000 mudas de teca, no sistema de semeadura em canteiro. As mudas produzidas neste sistema são conhecidas como "toco de raiz nua". Para o plantio de 1 ha de teca no espaçamento $3 \times 2 \mathrm{~m}$, serão necessárias 1.833 mudas (considerando replantio de $10 \%$ ), o que corresponde a um custo total de $\mathrm{R} \$ 189,70$ para essa atividade.

Nas operações de preparo do terreno para uma área de cerrado, os custos considerados foram: derrubada da vegetação, enleiramento, aração, gradagem e combate à formiga. Para o plantio, foram considerados os custos com o replantio, a adubação, a aplicação de calcário e o transporte, além dos custos com o próprio plantio das mudas.

Com relação à manutenção do plantio, no primeiro ano foram considerados o combate intensivo à formiga, o controle de plantas invasoras (capina), o transporte de mudas e a eliminação de brotações. As mudas do tipo "toco de raiz nua" normalmente emitem várias brotações, impedindo o desenvolvimento normal das mudas. Do segundo ao quarto ano, foram consideradas as atividades de combate à formiga, controle de plantas invasoras (roçada) e transporte de mão-de-obra. Vale salientar que, comumente, a atividade de controle de plantas invasoras (roçada) é realizada somente até o terceiro ano, sendo feita no quarto se considerada de extrema necessidade. $\mathrm{O}$ combate à formiga após o quarto ano é realizado também somente conforme a necessidade.

A partir do segundo ano, até a árvore atingir altura comercial $(8 \mathrm{~m})$, são realizadas desramas a um custo de $\mathrm{R} \$ 40,00 /$ ha. Adubações de manutenção com aplicação de $300 \mathrm{~kg}$ de NPK 4-20-20 + FTE Br12 por hectare ocorrem somente após os desbastes, a um custo médio de R $\$ 18,90 /$ ha. São comumente realizadas até o terceiro desbaste. Para determinação dos custos de manutenção anual de 1 ha de plantio de teca, foram consideradas as atividades de conservação de estradas, aceiros e cercas, vigilância, administração e custo da terra. $\mathrm{O}$ valor da terra considerado foi de $\mathrm{R} \$ 500,00 / \mathrm{ha}$, para uma taxa de juros de $6 \%$ ao ano.

Os valores dos custos da atividade de desbaste foram calculados em função da quantidade de madeira, em metro estéreo, retirada do plantio. $\mathrm{O}$ fator de cubicação foi 3,31 metros estéreo para cada metro cúbico de madeira desbastada. Foram considerados custos com marcação, abate, desgalhamento, traçamento, extração $(250 \mathrm{~m})$, carregamento, transporte e descarregamento no pátio de estocagem. Para o cálculo desses custos considerou-se o tempo gasto em cada atividade desenvolvida, tendo como base o preço da hora-máquina de cada equipamento e o preço da mão-de-obra utilizada. Assim como para os custos de desbaste, os custos de exploração foram calculados em função da quantidade de madeira, em metro estéreo, retirada do plantio, tendo sido considerados o mesmo fator de cubicação e as mesmas atividades.

\subsection{Estrutura de Receitas}

Para determinação das receitas desta análise econômica, considerou-se o programa de desbaste para a teca exposto no Quadro 2.

As receitas geradas pela venda da madeira ao longo da rotação de 25 anos encontram-se no Quadro 3. Estas foram calculadas de acordo com o volume de madeira explorado nos desbastes e no corte final. Os consumidores internacionais compram a madeira da teca em toras ou serrada, com preços variando de acordo com o diâmetro da tora e com a qualidade do produto. Nesta análise, não foi feita distinção de receitas para especificações de venda da madeira; as receitas geradas contemplaram todas as alternativas de comercialização.

Quadro 2 - Programa de desbaste de teca, para uma rotação de 25 anos

Table 2 - Teak thinning program over a 25-year-old rotation

\begin{tabular}{|c|c|c|c|}
\hline $\begin{array}{c}\text { Intensidade de } \\
\text { Desbaste (\%) }\end{array}$ & Desbaste & Ano & $\begin{array}{c}\text { oo de Árvore } \\
\text { Desbastada por } \\
\text { Hectare }\end{array}$ \\
\hline 50 & 1 & 4 & 833 \\
\hline 40 & 2 & 8 & 333 \\
\hline 40 & 3 & 12 & 200 \\
\hline 40 & 4 & 16 & 120 \\
\hline 40 & 5 & 20 & 72 \\
\hline 100 & CF & 25 & 108 \\
\hline Total & & & 1.666 \\
\hline
\end{tabular}

R. Árvore, Viçosa-MG, v.27, n.4, p.487-494, 2003 
Normalmente, as empresas encontram dificuldades para comercialização da madeira de primeiro desbaste, que apresenta cerca de $81,19 \%$ de alburno, embora suas propriedades sejam consideradas semelhantes a cernes de madeiras de grande valor comercial. Sua utilização atual se restringe à fabricação de brinquedos ou artesanatos de modo geral.

Com os dados de custos e receitas foi possível elaborar um fluxo de caixa para o plantio de teca submetido a desbastes, por período de ocorrência do item de custo e, ou, receita, como mostra o Quadro 4.

\subsection{Análise Econômica}

Para verificar a viabilidade econômica do plantio de teca submetido a desbastes, foram utilizados os seguintes critérios de análise econômica de projetos:

A) valor presente líquido - VPL, em que

$$
V P L=\sum_{j=1}^{n} R_{j}(1+i)^{-j}-\sum_{j=1}^{n} C_{j}(1+i)^{-j} ;
$$

B) benefício (custo) periódico equivalente - B(c)PE, em que

$$
\mathrm{BPE}=\frac{\operatorname{VPL}\left[(1+\mathrm{i})^{\mathrm{t}}-1\right](1+\mathrm{i})^{\mathrm{nt}}}{(1+\mathrm{i})^{\mathrm{nt}}-1}
$$

C) taxa interna de retorno - TIR, dada pela relação

$$
\sum_{j=1}^{n} R_{j}(1+T I R)^{-j}=\sum_{j=1}^{n} C_{j}(1+T I R)^{-j} ;
$$

D) valor esperado da terra - VET, em que

$$
\operatorname{VET}=\frac{R_{j}(1+i)^{t}}{(1+i)^{t}-1} .
$$

Os termos são: $\mathrm{R}_{\mathrm{j}}=$ receitas do projeto considerado; $\mathrm{C}_{\mathrm{j}}=$ custos no projeto considerado; $\mathrm{i}=$ taxa de desconto; $\mathrm{n}=$ duração do projeto; e $\mathrm{t}=$ número de períodos de capitalização.

A taxa de desconto escolhida foi de $10 \%$ ao ano, por ser uma das mais utilizadas pelo setor florestal brasileiro, que tradicionalmente trabalha com taxas entre 6 e $12 \%$, embora seja mais coerente a aplicação de taxas de longo prazo, mais condizentes com o tempo de maturação dos projetos, calculadas em função de seus fatores formadores, como afirma Júnior et al. (1997). Uma

R. Árvore, Viçosa-MG, v.27, n.4, p.487-494, 2003 análise de sensibilidade foi conduzida para detectar a sensibilidade dos critérios às variações na taxa de desconto e nos níveis de custos e receitas.

Quadro 3 - Receitas obtidas com a venda da madeira de teca

Table 3 -Income obtained from the sale of teak wood

\begin{tabular}{|c|c|}
\hline Idade de Desbaste (anos) & Receita (R\$/ha) \\
\hline 04 & $3.250,00$ \\
\hline 08 & $7.816,80$ \\
\hline 12 & $14.233,95$ \\
\hline 16 & $17.564,40$ \\
\hline 20 & $26.231,95$ \\
\hline Corte final & $76.542,00$ \\
\hline Total & $145.639,10$ \\
\hline
\end{tabular}

Quadro 4 - Fluxo de caixa para o plantio de teca subme-

\begin{tabular}{|c|c|c|}
\hline $\begin{array}{l}\text { Período de } \\
\text { Ocorrência }\end{array}$ & Custo (R\$/ha) & Receita ( $\mathrm{R} \$ / \mathrm{ha})$ \\
\hline 0 & $1.035,1$ & 0 \\
\hline 1 & 348,0 & 0 \\
\hline 2 & 324,0 & 0 \\
\hline 3 & 324,0 & 0 \\
\hline 4 & $6.708,1$ & $3.250,00$ \\
\hline 5 & 172,5 & 0 \\
\hline 6 & 132,5 & 0 \\
\hline 7 & 132,5 & 0 \\
\hline 8 & $9.588,6$ & $7.816,80$ \\
\hline 9 & 132,5 & 0 \\
\hline 10 & 132,5 & 0 \\
\hline 11 & 132,5 & 0 \\
\hline 12 & $10.069,2$ & $14.233,95$ \\
\hline 13 & 132,5 & 0 \\
\hline 14 & 132,5 & 0 \\
\hline 15 & 132,5 & 0 \\
\hline 16 & $8.826,1$ & $17.564,40$ \\
\hline 17 & 132,5 & 0 \\
\hline 18 & 132,5 & 0 \\
\hline 19 & 132,5 & 0 \\
\hline 20 & $6.833,2$ & $26.231,95$ \\
\hline 21 & 132,5 & 0 \\
\hline 22 & 132,5 & 0 \\
\hline 23 & 132,5 & 0 \\
\hline 24 & 132,5 & 0 \\
\hline 25 & $13.137,5$ & $76.542,00$ \\
\hline
\end{tabular}
tido a desbastes

Table 4 - Cashflow for teak plantation submitted to thinning 


\section{RESULTADOS E DISCUSSÃO}

\subsection{Valor Presente Líquido (VPL)}

A viabilidade econômica do plantio de teca submetido à desbastes pelo método do valor presente líquido (VPL) é indicada pela diferença positiva entre receitas e custos, atualizados de acordo com a taxa de desconto de $10 \%$ ao ano. A Figura 1 mostra a curva do VPL de acordo com as variações na taxa de desconto. Observe que para a taxa de desconto de $10 \%$ ao ano, utilizada nesta avaliação, o VPL obtido foi de $\mathrm{R} \$ 6.270,32 / \mathrm{ha}$ e que, como esperado, ele decresce à medida que essa taxa aumenta. É possível verificar que o VPL será positivo até uma taxa de desconto de $15 \%$ (VPL $=\mathrm{R} \$ 67,72)$, dando segurança a este investimento (plantio). Taxas de desconto maiores que esta inviabilizam o plantio, sendo então melhor investir em outras alternativas potencialmente mais rentáveis.

A Figura 2 apresenta a análise de sensibilidade do VPL sobre as variações das receitas e dos custos.

Analisando a Figura 2, nota-se que mesmo uma redução de $20 \%$ sobre as receitas não tornaria o plantio inviável, ou seja, caso ocorra uma queda de $20 \%$ no preço da madeira de teca o plantio ainda apresentaria um valor presente líquido positivo (VPL $=\mathrm{R} \$ 1.232,71 / \mathrm{ha}$ ).

Por outro lado, esse comportamento evidencia que o plantio de teca, conforme as condições estabelecidas, é um investimento que apresenta margem de segurança baixo. No entanto, considerando que a demanda pela madeira dessa espécie é maior que a oferta no mercado exterior, é pouco provável, a curto prazo, uma queda repentina no preço da madeira, de tal forma que inviabilize o plantio da teca.

Quanto à análise de sensibilidade do VPL em relação ao custo, é possível verificar que o aumento de até $30 \%$ não inviabiliza o plantio (VPL $=\mathrm{R} \$ 595,01 / \mathrm{ha})$. $\mathrm{O}$ alto valor da madeira de teca é suficiente para garantir rentabilidade ao plantio nessa condição.

\subsection{Benefício (Custo) Periódico Equivalente [B(C)PE]}

O critério benefício (custo) periódico equivalente [B(c)PE] converte os fluxos de caixa líquidos em uma série equivalente de valores iguais e, geralmente, anuais. A Figura 3 mostra a curva do B(c)PE de acordo com as variações na taxa de desconto.
$\mathrm{O} \mathrm{B}(\mathrm{c}) \mathrm{PE}$ apresentou resultado semelhante ao encontrado pelo VPL, ou seja, o B(c)PE também será positivo somente até uma taxa de desconto de $15 \%$. Para a taxa de desconto utilizada nesta avaliação (10\% ao ano), o B(c)PE obtido foi de R \$690,79/ha/ano, viabilizando o plantio da teca.

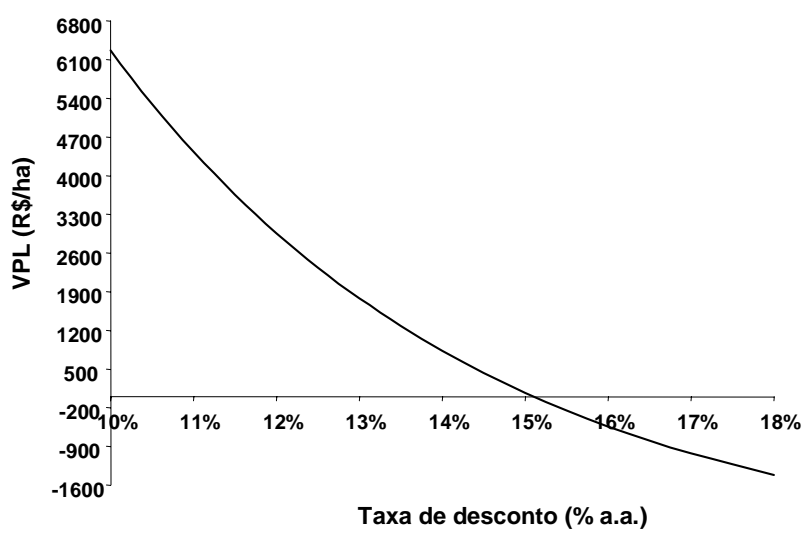

Figura 1 - Curva do valor presente líquido (VPL) de acordo com as variações na taxa de desconto.

Figure 1 - Net Present Value (NPV) curve based on discount rate variations.
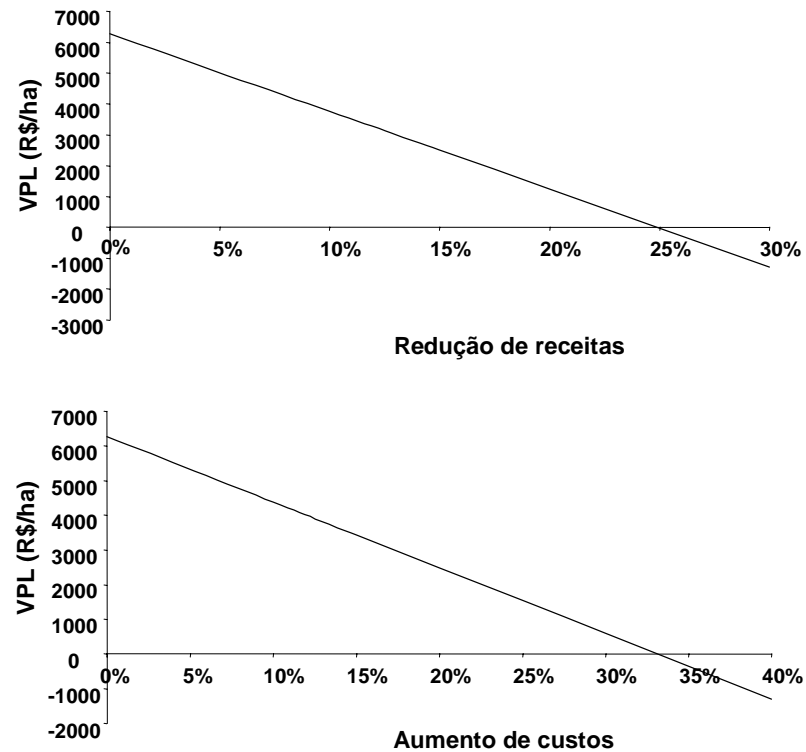

Figura 2 - Análise de sensibilidade do valor presente líquido (VPL) às variações de redução de receitas e de aumento de custos.

Figure 2 - Net Present Value (NPV) sensibility analysis, based on income reduction and cost increase variations.

R. Árvore, Viçosa-MG, v.27, n.4, p.487-494, 2003 


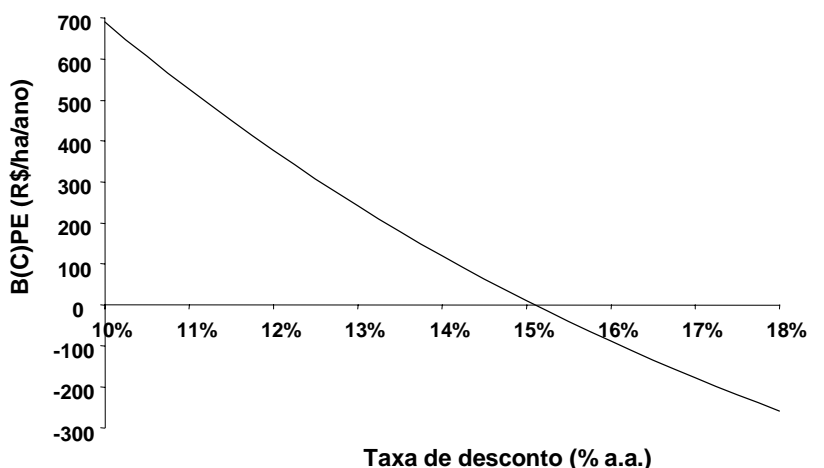

Figura 3 - Curva do benefício (custo) periódico equivalente [B(c)PE] de acordo com as variações na taxa de desconto. Figure 3 - Equivalent Annual Value (EAV) curve based on discount rate variations.

Com relação à análise de sensibilidade para as variações de receita e custos, o critério B(c)PE inviabiliza o plantio com redução de $25 \%$ das receitas e $35 \%$ de aumento dos custos.

\subsection{Taxa Interna de Retorno (TIR)}

A taxa interna de retorno (TIR) de um projeto, também chamada de eficiência marginal do capital, é a taxa de desconto que iguala o valor atual das receitas futuras ao valor atual dos custos futuros do projeto, ou seja, é a taxa média de crescimento de um investimento.

A TIR do presente investimento foi de $15,1 \%$ ao ano, como mostra a Figura 4. Esse valor é semelhante aos determinados em avaliações econômicas de plantações de eucalipto, como a avaliação feita por Silva et al.

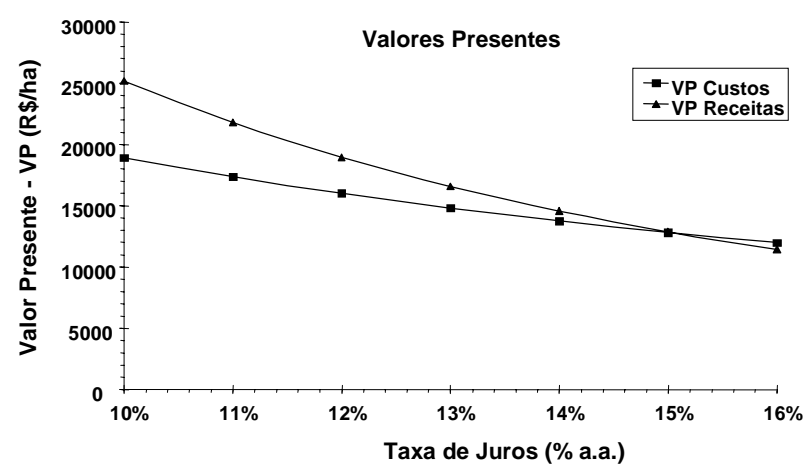

Figura 4 - Taxa interna de retorno (TIR) do plantio de teca submetido a desbastes.

Figure 4 - Internal Rate of Return (IRR) of the teak plantation submitted to thinning.

(1997) de um monocultivo de Eucalyptus grandis W. Hill ex Maiden, em que a TIR foi de $14 \%$ ao ano.

Pela TIR do presente estudo, o plantio de teca também seria viável, considerando que a taxa mínima de atratividade é de $10 \%$ ao ano. Vale salientar que, neste caso, as decisões de aceitação ou rejeição do projeto são perfeitamente coincidentes com aquelas obtidas quando se aplica o método do VPL, dado à independência do projeto.

O valor encontrado para a TIR demonstra que as receitas descontadas seriam superiores aos custos descontados, mesmo se o mercado trabalhasse com taxas superiores a $10 \%$ ao ano, até o limite de $15,1 \%$ ao ano.

O comportamento da TIR mediante as variações de receitas e custos pode ser observado na Figura 5. Note
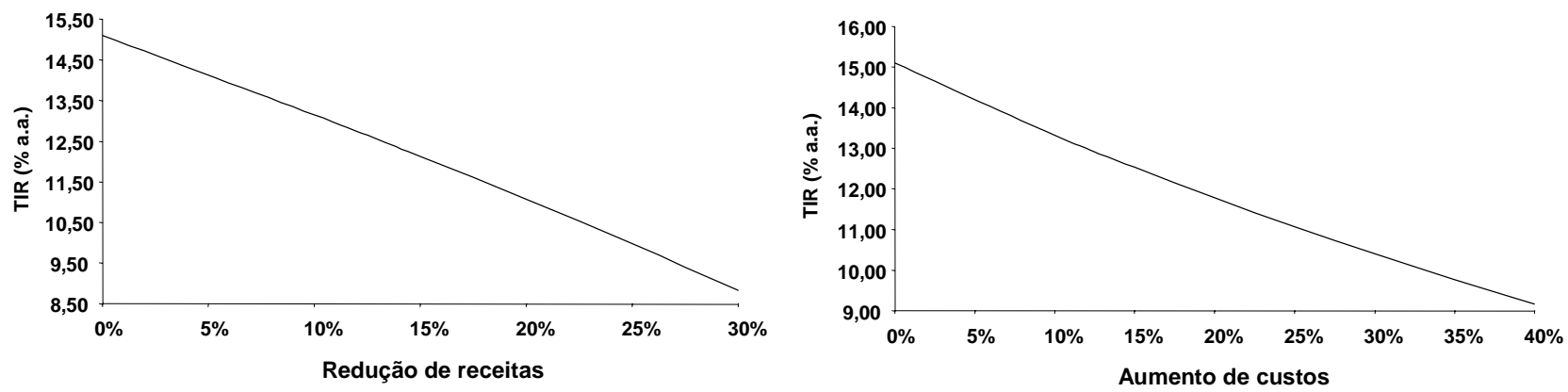

Figura 5 - Comportamento da taxa interna de retorno (TIR) mediante as variações de redução de receitas e de aumento de custos.

Figure 5 - Internal Rate of Return (IRR) behavior due to income reduction and cost increase variations. 
que para uma redução de $25 \%$ das receitas a TIR é de 9,98\% ao ano, abaixo da taxa de remuneração alternativa do capital (TMA $=10 \%$ ao ano). Já para um aumento de $35 \%$ dos custos a TIR é de $9,77 \%$ ao ano. Nessas condições, o projeto não seria viável economicamente. Por outro lado, considerando uma alternativa segura de aplicação, como no caso da poupança, mas que atualmente apresenta rendimento muito baixo (aproximadamente $8,4 \%$ a.a.), optar pelo plantio de teca seria mais rentável para o investidor.

\subsection{Valor Esperado da Terra (VET)}

O cálculo do valor esperado da terra (VET) baseiase na receita líquida perpétua (RT - CT), excluindo-se o custo da terra, a ser obtida do plantio da teca. Para a taxa de $10 \%$ ao ano, o VET calculado foi de R $\$ 7.240,94 /$ ha, como se observa na Figura 6, que mostra também o valor do VET para diferentes taxas de desconto. Esse valor é o preço máximo de compra da terra nua, considerando uma série infinita de rotações, a ser utilizada para produção de madeira de teca, conforme as condições estabelecidas na análise. Quando se trabalha com uma taxa de desconto de $15 \%$ ao ano o plantio torna-se inviável economicamente, porque o VET de $\mathrm{R} \$ 300,79 /$ ha é menor do que o valor da terra considerado nesta análise $(\mathrm{R} \$ 500,00 / \mathrm{ha})$.

A análise de sensibilidade mostrou que o projeto será viável mesmo com a redução de $20 \%$ nas receitas e o aumento de $30 \%$ nos custos, como mostra a Figura 7. Nessas condições, o VET foi de R\$1.691,11/ha e $\mathrm{R} \$ 1.088,47 / \mathrm{ha}$, respectivamente.

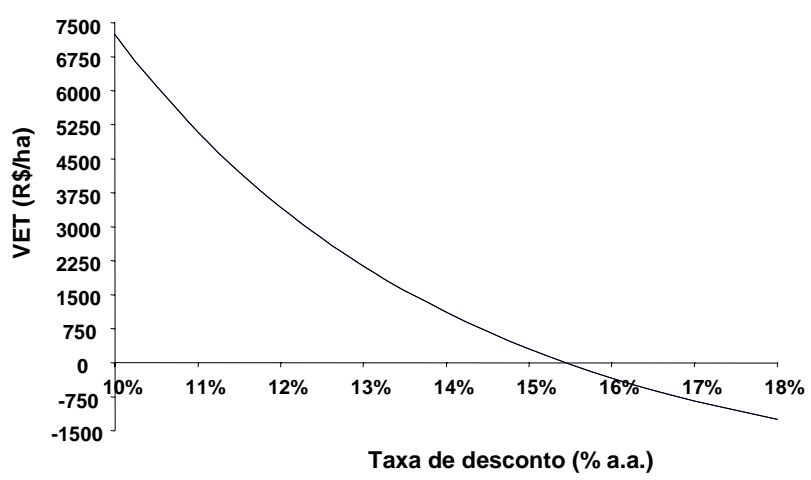

Figura 6 - Curva do valor esperado da terra (VET) de acordo com as variações na taxa de desconto.

Figure 6 - Land expected value (LEV) based on discount rate variations.
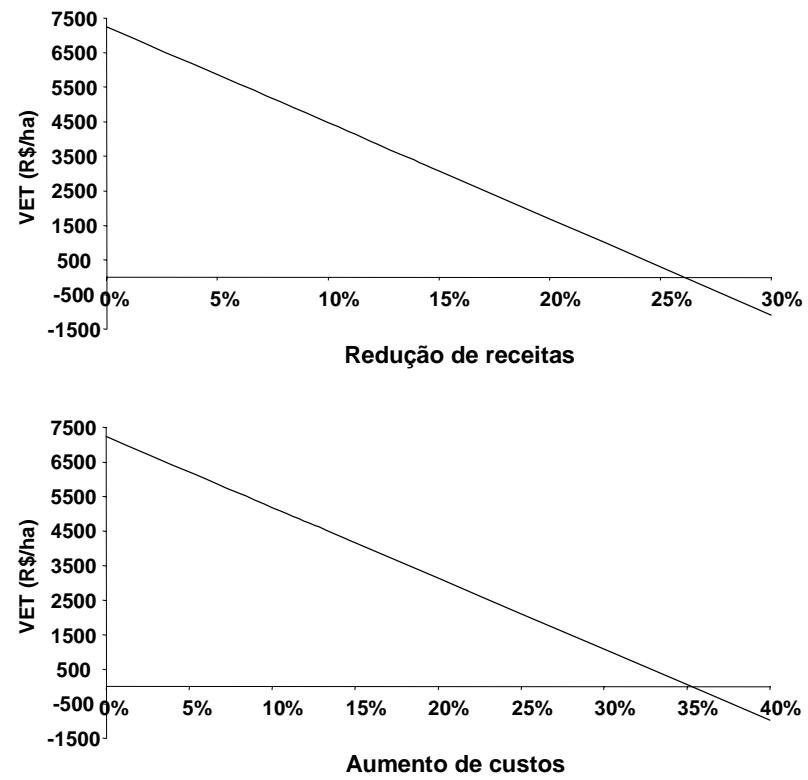

Figura 7 - Comportamento do valor esperado da terra (VET) mediante as variações de redução de receitas e de aumento de custos.

Figure 7 - Land expected value (LEV) behavior due to income reduction and cost increase variations.

\section{CONCLUSÃO}

- O plantio de teca, para as condições estabelecidas, foi considerado viável por todos os indicadores utilizados nesta análise econômica.

- As reduções nas receitas, com a queda do preço da madeira de teca, não podem ser de $25 \%$, mantidos outros fatores da análise, sob pena de inviabilizar o plantio.

- O aumento de $35 \%$ sobre os custos considerados nesta análise inviabilizaria o plantio da teca, mantidos constantes outros fatores da análise.

- O plantio da teca é mais sensível às variações na redução da receita do que às variações no aumento dos custos, o que é devido ao fato de o preço da madeira ser muito alto no mercado.

\section{REFERÊNCIAS BIBLIOGRÁFICAS}

FINGER, Z; FINGER, F. A.; DRESCHER, R. Teca (Tectona grandis L.f.): plante esta idéia. In: SIMPÓSIO BRASILEIRO DE PÓS-GRADUAÇÃO EM ENGENHARIA FLORESTAL, 1., 2001, Santa Maria-RS. Anais... Santa Maria: UFSM, 2001. CD-Rom.

R. Árvore, Viçosa-MG, v.27, n.4, p.487-494, 2003 
JÚNIOR, V. B. L.; REZENDE, J. L. P.; OLIVEIRA, A. D. Determinação da taxa de desconto a ser usada na análise econômica de projetos florestais. Revista Cerne, v. 3, n. 1, p. 45-66, 1997.

RONDON NETO, R. M.; MACEDO, R. L. G.;

TSUKAMOTO FILHO, A. A. Formação de povoamentos florestais com Tectona grandis L.f. (Teca). Boletim

Técnico - Série Extensão, v. 7, n. 33, p. 1-29, 1998.
SILVA, M. L. et al. Viabilidade econômica do

reflorestamento do eucalipto consorciado com a cultura do feijão: um estudo de caso. Revista Árvore, v. 21, n. 4, p. $527-535,1997$.

VEIT, L. F. Dinheiro não cresce em árvores. Revista Silvicultura, n. 83, p. 38-39, 2000. 